\title{
Mechanisms of color constancy
}

\author{
M. D'Zmura and P. Lennie \\ Center for Visual Science and Department of Psychology, University of Rochester, Rochester, New York 14627 \\ Received April 21, 1986; accepted June 27, 1986

\begin{abstract}
We develop a model of how the visual system finds the colors of objects that have unknown shapes and positions. The model relies on mechanisms of light adaptation, coupled with eye movements, to recover three descriptors of surface reflectance that are represented in the signals of an achromatic mechanism and two color-opponent mechanisms. These descriptors are transformed to yield estimates of hue, the dimension of surface color that is independent of object shape and viewing geometry.
\end{abstract}

\section{INTRODUCTION}

The intrinsic chromatic properties of objects are given by surface reflectance functions, which specify the proportion of incident illumination reflected by a surface as a function of wavelength. Recovery of surface reflectance functions from the lights that reach our eyes is a complex task, because the reflected light is a composite signal: it depends on both surface reflectance and the spectral power distribution (SPD) of the illuminant. Mechanisms that provide color constancy must decompose this signal and discount the spectral properties of the illuminant in order to establish the colors of objects in terms of their surface reflectance functions.

A trichromatic visual system can represent a surface reflectance function using at most three parameters. Similarly, it must represent illuminants by at most three parameters, so unless surface reflectances and illuminants can be represented adequately by only three variables, color constancy will be impossible. Fortunately, three-dimensional descriptions of surfaces and illuminants are adequate because there is an underlying simplicity in natural chromatic signals. Principal components analyses of daylight ${ }^{1,2}$ and of surface reflectances ${ }^{3}$ suggest that much of the variance in each of these domains may be accounted for by a small set of components: only three components added together in appropriate proportions are required to represent any phase of daylight, and three to five appropriately weighted components are required for the reconstruction of most surface reflectances. ${ }^{4}$ This simplicity is the key to color constancy and has been exploited in the formal solutions offered by Sällström, ${ }^{5}$ Brill,,$^{6,7}$ Buchsbaum, ${ }^{8}$ and Maloney and Wandell. ${ }^{4,9}$ The purpose of this paper is to extend these formal treatments in two ways.

For adequate color constancy the perceived color of an object should be stable not only through changes in the SPD of the light that falls on a scene but also through changes in the position of the object, the illuminant, and the viewer. Because surface reflectance functions depend not only on material properties but also on geometric variables, the lights that reach the eyes from an object change when viewing geometry is changed. Even if the viewing geometry is held fixed, the spectral distribution of the light from a solid object generally varies from point to point across its surface.
Our first aim is to show how the hue of an object can be rendered independently of its shape and position. We show formally how a trichromatic system that can find the SPD of an illuminant can discount the illuminant and find the completely stable hues of objects without discarding the geometric information that is carried by the dimensions of lightness and saturation.

The second aim of the paper is to develop a physiologically plausible account of the mechanisms that might undertake the computation. We show first how mechanisms of light adaptation, both in the separate cone pathways and at opponent sites, can find and discount an illuminant. We then show how the signals from second-stage mechanisms can be transformed to yield a stable hue descriptor.

\section{SURFACE REFLECTANCE AND THE STABILITY OF HUE}

We follow Yilmaz, ${ }^{10}$ Sällström, ${ }^{5}$ Brill, ${ }^{6,7}$ Buchsbaum, ${ }^{8}$ and Maloney and Wandell ${ }^{4,9}$ in using a small set of basis functions to provide an approximate representation of surface reflectance. For expository purposes we use the three basis functions shown in Fig. 1A. These are functions of wavelength; when added together in the correct proportions they describe the fraction of incident light reflected by a surface. The function $S_{1}(\lambda)$ is a constant function of wavelength; variations in the contribution of $S_{1}(\lambda)$ to surface reflectance represent variations along a "light-dark" dimension. The function $S_{2}(\lambda)$ represents variation along a "red-green" dimension, while the function $S_{3}(\lambda)$ represents variation along a "yellow-blue" dimension. These functions are the first three terms in a Fourier series expansion and have been chosen for their simplicity. The functions that best describe any particular class of surfaces will be the set that accounts for the greatest proportion of the variance in the collection of surface reflectances. For natural surfaces these are probably not the functions shown in Fig. 1A, but they would share the same general features. ${ }^{4}$

The two "color" functions $S_{2}(\lambda)$ and $S_{3}(\lambda)$ have negative as well as positive values and cannot, by themselves, describe a real surface reflectance; they are used only in combination with the "light-dark" function $S_{1}(\lambda)$. Figure 2 shows how any surface reflectance function can be approximately reconstituted by adding together appropriate amounts of 

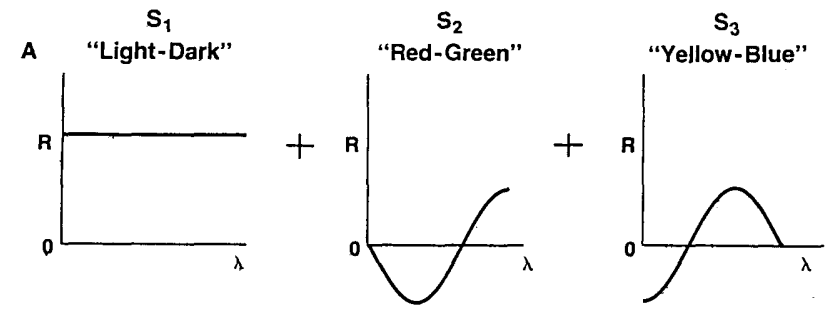

$S_{0}$

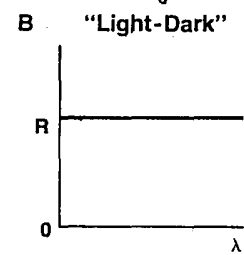

Fig. 1. A, Three basis functions $S_{1}(\lambda), S_{2}(\lambda)$, and $S_{3}(\lambda)$ used to model surface reflectance functions. The absolute values of reflectance $(R)$ for $S_{1}, S_{2}$, and $S_{3}$ are not indicated; their contributions to a given surface reflectance are set by scalars in the model. $B$, The basis function $S_{0}(\lambda)$, identical to $S_{1}(\lambda)$, that describes the spectral properties of the specular component of reflectance. Part A of this figure may be reinterpreted as a description of the spectral properties of the diffuse component.
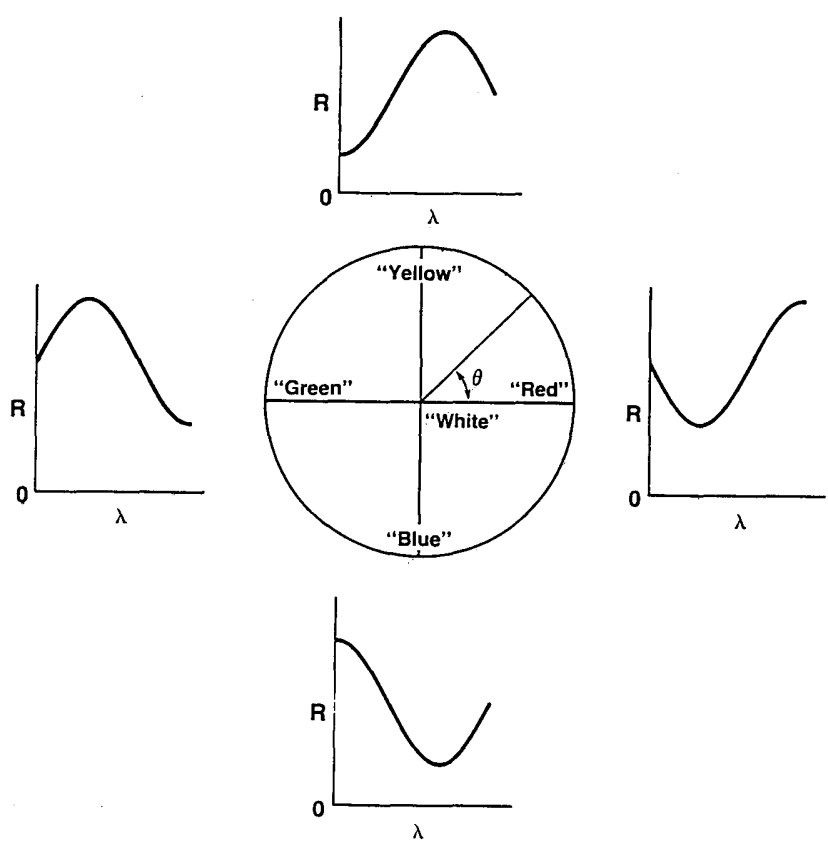

Fig. 2. Color circle showing variation in contributions of $S_{1}, S_{2}$, and $S_{3}$ to surface reflectance functions. The basis functions are summed in appropriate ways to generate the surface reflectance functions shown here. The ratio of contributions to reflectance made by $S_{3}$ and $S_{2}$ determines the azimuth $\theta$, which represents hue.

these functions. For instance, by adding the "red-green" function $S_{2}(\lambda)$ to the "light-dark" function $S_{1}(\lambda)$, the result is a "red" reflectance function, while by subtracting $S_{2}(\lambda)$ from $S_{1}(\lambda)$ we arrive at a "green" reflectance function.

\section{Color at a Point}

We do not know how the psychological dimensions of color are linked to surface reflectance, but a consistent account is suggested by the color circle of Fig. 2.10 Saturation is related to the proportion of "colored" light to "white" light and so is related to the amounts $n_{2}$ and $n_{3}$ contributed by $S_{2}(\lambda)$ and $S_{3}(\lambda)$ relative to the amount $n_{1}$ contributed by $S_{1}(\lambda)$. Bearing in mind that the values of $n_{2}$ and $n_{3}$ may be negative; saturation is related to the ratio $\left(n_{2}^{2}+n_{3}^{2}\right)^{1 / 2} / n_{1}$. The hue of a surface is related to the ratio $n_{3} / n_{2}$ of the two "color" terms: the amount of "yellow-blue" relative to the amount of "redgreen." If hue is taken to be the azimuthal angle measured from the "red" half-axis, as shown in Fig. 2, then hue is given by $\arctan \left(n_{3} / n_{2}\right)$. The lightness of a surface is related to the amount $n_{1}$ of the flat component $S_{1}(\lambda)$ of a given reflectance function.

Formally, a surface reflectance function at a point on the surface of an object is given as the following linear combination:

$$
S(\lambda) \simeq \sum_{i=1}^{3} n_{i} S_{i}(\lambda)
$$

\section{Surface Reflectances of Solid Objects}

The problem with the model of reflectance given by formula (1), which is used with minor variations in most formal approaches to color constancy, ${ }^{4-9}$ is that it does not adequately describe variations in reflectance that depend on geometric variables. The model may be extended to cover the dependence on geometry by recognizing that the "lightdark" contribution to surface reflectance can come from two physically distinct components. The first of these is the specular component, which arises at the optical interface between air and the surface ${ }^{11-14}$; the second is the diffuse component, which arises from the absorption and scattering of light by molecules underlying the surface. ${ }^{11,14-16}$

Figure 1B indicates that the specular component $S_{0}(\lambda)$ of most objects is essentially flat across the visible spectrum. ${ }^{17}$ The specular reflection therefore has the relative SPD of the illuminant.

At any point on the surface of a uniformly colored object, the total reflectance is the sum of the specular and diffuse components. For such an object (e.g., a green pepper) the contributions of these two terms to total reflectance vary from point to point. Figure $3 \mathrm{~A}$ depicts this variation in a space that represents the quantum catches in the three classes of cones. Responses to the lights from different points on the surface of the object fall along the locus $L$. Quantum catches from the specular component alone lie along the axis $I$ that describes the illuminant, while quantum catches from the diffuse component alone lie along the axis D.

Where the specular component dominates the total reflectance, for instance in a "highlight" region, the surface appears light and desaturated and the trichromatic response lies close to the axis $I$ defined by the illuminant. The surface appears desaturated at such points because the color contribution of the diffuse component is small relative to the lightness contribution from the specular component. Points lying outside highlights are typically darker and more saturated and give rise to responses that lie closer to the axis $D$, which describes the diffuse component.

Since the response to the light from any point on a uniformly colored surface is a sum of responses to specular and diffuse components, the locus $L$ of responses to different points lies in the plane defined by the axes $D$ and $I$, as noted 

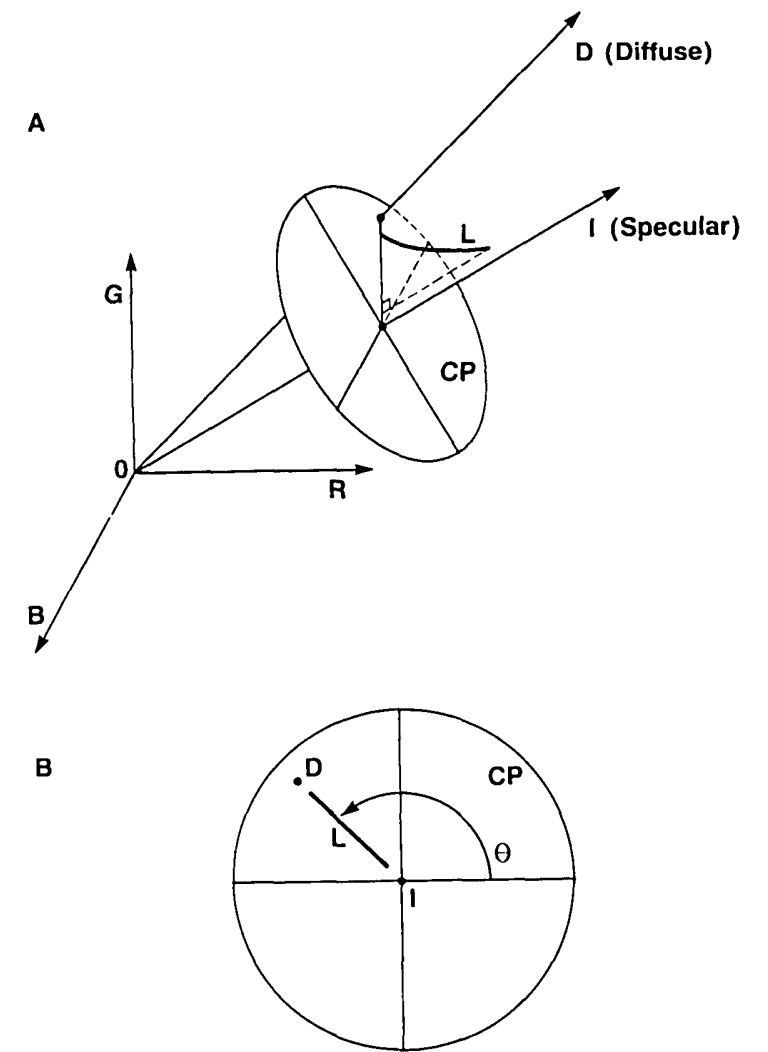

Fig. 3. A, A three-dimensional space of photoreceptor quantum catches (R, G, B) in which lies the locus $L$ of responses to lights from different points on a single, uniformly colored object; both specular and diffuse components of reflectance vary across the surface of the object. $L$ lies within a single plane determined by the response to the specular component, which has the chromaticity of the illuminant and varies along the axis labeled $I$, and the response to the diffuse component, which varies along the axis labeled $D$. A chromaticity plane $C P$ is centered on and perpendicular to the axis $I$. Variations in $L$ perpendicular to $C P$ represent changes in lightness. $\mathrm{B}$, Projection of $L$ onto $C P$, showing that saturation may also change across a single uniformly colored surface. The azimuth $\theta$ that represents hue is a geometrically stable color descriptor.

by Shafer. ${ }^{17}$ We use this fact later to show how the chromaticity of the illuminant can be found by using highlights.

The plane CP of Fig. 3, which is centered on and perpendicular to the axis that describes the illuminant, is a chromaticity plane. This is easiest to imagine if the illuminant is white. Figure $3 \mathrm{~B}$ shows that responses to all the lights from a uniformly colored surface lie along a line segment in the plane $C P$ that joins the chromaticity of the illuminant to the chromaticity of the diffuse component. This line spans the range of possible color signals from the surface: at one extreme $(I)$ the light received from a point on a surface arises entirely from the specular component, while at the other extreme $(D)$ it arises entirely from the diffuse component.

The variation in specular and diffuse components of reflectance from point to point on the surface results in corresponding variations in lightness and saturation. Only the ratio of contributions from "yellow-blue" and "red-green" basis functions is constant across a uniformly colored surface: although the contributions of these two chromatic terms to reflectance may vary across a solid object, the contributions vary identically in a way that is determined by the intensity of the diffuse component of reflectance. This stable hue is represented in Fig. $3 \mathrm{~B}$ by the azimuth $\theta$.

\section{Formal Expression of Surfacè Reflectance}

The concepts that have been outlined graphically are formalized by expressing the surface reflectance $S(\mathbf{x}, \lambda)$ at a point $\mathbf{x}=\left(x_{1} x_{2}\right)$ on a uniformly colored object as the following sum:

$$
\begin{aligned}
S(\mathbf{x}, \lambda) & \simeq f_{s}(\mathbf{x}) S_{0}(\lambda)+f_{d}(\mathbf{x})\left[\sum_{i=1}^{3} n_{i} S_{i}(\lambda)\right] \\
& =\text { "specular" + "diffuse". }
\end{aligned}
$$

The function $f_{s}(\mathbf{x})$ describes the variation in the intensity of the specular component with location on the surface, while the function $f_{d}(\mathbf{x})$ describes the corresponding variation in the intensity of the diffuse component.

Because the basis functions $S_{0}(\lambda)$ and $S_{1}(\lambda)$ are identical, the two terms that govern their intensities may be combined into a single term $f_{L}(\mathbf{x})$ that represents "lightness":

$$
f_{L}(\mathbf{x})=f_{s}(\mathbf{x})+f_{d}(\mathbf{x}) n_{1} .
$$

Formula (2) may be recast to express surface reflectance as the sum of a "lightness" term and two "color" terms:

$$
\begin{aligned}
S(\mathbf{x}, \lambda) & \simeq f_{L}(\mathbf{x}) S_{1}(\lambda)+f_{d}(\mathbf{x}) n_{2} S_{2}(\lambda)+f_{d}(\mathbf{x}) n_{3} S_{3}(\lambda) \\
& =\text { "lightness" + "red-green" + "yellow-blue". }
\end{aligned}
$$

The geometric stability of hue may now be specified more precisely: hue is related to the arctangent of the ratio of the terms that describe the contributions of $S_{3}(\lambda)$ and $S_{2}(\lambda)$ to a given reflectance. In forming the ratio $f_{d}(\mathbf{x}) n_{3} / f_{d}(\mathbf{x}) n_{2}$, the geometry-dependent term $f_{d}(\mathbf{x})$ drops out. The resulting ratio, $n_{3} / n_{2}$, depends solely on material properties.

The linear model of reflectance expressed in formula (4) suggests that color constancy depends on the accurate recovery of the terms $f_{L}, f_{d} n_{2}$, and $f_{d} n_{3}$, which are the weights on the "light-dark," "red-green," and "yellow-blue" basis functions that describe the reflectance of a surface. These weights depend on geometric factors: they vary from point to point on a surface according to the relative strengths of the specular and diffuse components of reflectance. If we know $f_{L}(\mathbf{x})$ and $f_{d}(\mathbf{x})$ we can compute the numbers $n_{1}, n_{2}$, and $n_{3}$, which depend solely on material properties. If we do not .know $f_{L}(\mathbf{x})$ and $f_{d}(\mathbf{x})$, the only geometrically stable descriptor that can be extracted is hue.

\section{DISCOUNTING A KNOWN ILLUMINANT}

The description of surface reflectance given by $f_{L}, f_{d} n_{2}$, and $f_{d} n_{3}$ can be extracted only if the spectral properties of the illuminant can be discounted. There are well-developed formal solutions to the problem of discounting a known illuminant 4,8 ; these rely on our ability to represent the SPD's of illuminants using a small set of basis functions. As a result, an illuminant may be discounted by applying an appropriate linear transformation to quantum catch signals. In what follows, we show that Buchsbaum's 8 method for discounting the illuminant can be applied to scenes in which surfaces are described by formula (4). 


\section{Approximate Representation of Illuminants}

By analogy to formula (1), which deals with surface reflectance, the SPD $I(\lambda)$ of a given illuminant is expressed as a sum of appropriate amounts of three basis functions $I_{1}(\lambda)$, $I_{2}(\lambda)$, and $I_{3}(\lambda)$ :

$$
I(\lambda) \simeq \sum_{i=1}^{3} m_{i} I_{i}(\lambda)
$$

If the basis functions $I_{i}(\lambda)$ are known, then illuminants that can be characterized by formula (5) are described fully by the numbers $m_{1}, m_{2}$, and $m_{3}$. Formula (5) is known to provide an adequate description of the phases of daylight. ${ }^{1,2}$

\section{Interaction of Illuminants and Reflectances}

Illuminants interact multiplicatively with surface reflectances to produce the reflected light. Because the specular component of reflectance is flat, specular reflection has the relative $\mathrm{SPD}$ of the illuminant, and so therefore does the light that penetrates the surface to interact with colorants.

The light $L(\mathbf{x}, \lambda)$ reflected by a uniformly colored surface is thus found by multiplying the reflectance $S(\mathbf{x}, \lambda)$ expressed in formula (4) by the illuminant $I(\lambda)$ of formula (5); suppressing dependence on wavelength, the result is

$$
\begin{aligned}
L(\mathbf{x}) \simeq & f_{L}(\mathbf{x})\left(\sum_{i=1}^{3} m_{i} I_{i} S_{1}\right) \\
& +f_{d}(\mathbf{x}) n_{2}\left(\sum_{i=1}^{3} m_{i} I_{i} S_{2}\right) \\
& +f_{d}(\mathbf{x}) n_{3}\left(\sum_{i=1}^{3} m_{i} I_{i} S_{3}\right) .
\end{aligned}
$$

Formula (6) makes evident the problem of discounting the illuminant. Consider the case in which the illuminant $I(\lambda)$ is spectrally flat. The three chromatic components in the reflected light $L$ will represent precisely the contributions made by each of the basis functions for surface reflectance; it is a straightforward matter to form three mechanisms, e.g., an achromatic mechanism and two color-opponent mechanisms, that combine photoreceptoral signals linearly so as to signal $f_{L}, f_{d} n_{2}$, and $f_{d} n_{3}$, respectively. However, when we change the SPD of the illuminant, we change the spectral distributions of the three components of the light $L$ that correspond to the three basis functions for reflectance, so mechanisms set up to discount "white" illumination will no longer abstract the terms $f_{L}, f_{d} n_{2}$, and $f_{d} n_{3}$.

\section{Decomposing the Color Signal}

The computational solution to this problem is evident in formula (6). If one can find the three numbers $m_{i}$ that describe the illuminant, then one can find the components in the light $L$ that correspond to the three basis functions for surface reflectance. One can then calculate how the sensitivities of mechanisms that abstract reflectance descriptors must change in response to a change in illumination.

The spectral sensitivities of the three photoreceptors are termed $Q_{1}(\lambda), Q_{2}(\lambda)$, and $Q_{3}(\lambda)$, and their respective quantum catches are $q_{1}, q_{2}$, and $q_{3}$. We have in mind broad sensitivities of the sort derived by Smith and Pokorny for human cones. ${ }^{18}$ The quantum catch $q_{k}$ of the $k$ th photoreceptor type with spectral sensitivity $Q_{k}(\lambda)$ from light $L(\lambda)$ is given by the integral of the product of $L(\lambda)$ and $Q_{k}(\lambda)$ over the visible spectrum. We employ the "inner-product" notation $\langle$,$\rangle as a shorthand for these integrals of products over$ the visible spectrum and continue to suppress dependence on wavelength:

$$
q_{k}=\left\langle L, Q_{k}\right\rangle=\int_{400}^{700} L(\lambda) Q_{k}(\lambda) \mathrm{d} \lambda .
$$

If the expression for reflected light $L$ in formula (6) is now substituted for $L$ in Eq. (7), then the result is that

$$
\begin{aligned}
q_{k}(\mathbf{x}) \simeq & f_{L}(\mathbf{x})\left\langle\sum_{i=1}^{3} m_{i} I_{i} S_{1}, Q_{k}\right\rangle \\
& +f_{d}(\mathbf{x}) n_{2}\left\langle\sum_{i=1}^{3} m_{i} I_{i} S_{2}, Q_{k}\right\rangle \\
& +f_{d}(\mathbf{x}) n_{3}\left\langle\sum_{i=1}^{3} m_{i} I_{i} S_{3}, Q_{k}\right\rangle, \quad \text { for } 1 \leq k \leq 3 .
\end{aligned}
$$

The terms in the brackets are integrals that specify the extent to which each basis function for surface reflectance contributes to the quantum catch of a given photoreceptor, given the illuminant described by the parameters $m_{i}$. There are three integrals per photoreceptor type, giving a total of nine. The numbers $f_{L}, f_{d} n_{2}$, and $f_{d} n_{3}$ remain the same for each photoreceptor type, and so expression (8) may be reformulated by using vectors and matrices. By writing the illuminant as a function of the vector $\mathbf{m}=\left(m_{1} m_{2} m_{3}\right)$ we find that

$$
\begin{aligned}
& {\left[\begin{array}{l}
q_{1}(\mathbf{x}) \\
q_{2}(\mathbf{x}) \\
q_{3}(\mathbf{x})
\end{array}\right]} \\
& \simeq\left[\begin{array}{c}
\left\langle I(\mathbf{m}) S_{1}, Q_{1}\right\rangle\left\langle I(\mathbf{m}) S_{2}, Q_{1}\right\rangle\left\langle I(\mathbf{m}) S_{3}, Q_{1}\right\rangle \\
\left\langle I(\mathbf{m}) S_{1}, Q_{2}\right\rangle\left\langle I(\mathbf{m}) S_{2}, Q_{2}\right\rangle\left\langle I(\mathbf{m}) S_{3}, Q_{2}\right\rangle \\
\left\langle I(\mathbf{m}) S_{1}, Q_{3}\right\rangle\left\langle I(\mathbf{m}) S_{2}, Q_{3}\right\rangle\left\langle I(\mathbf{m}) S_{3}, Q_{3}\right\rangle
\end{array}\right]\left[\begin{array}{l}
f_{L}(\mathbf{x}) \\
f_{d}(\mathbf{x}) n_{2} \\
f_{d}(\mathbf{x}) n_{3}
\end{array}\right] .
\end{aligned}
$$

The result is that the surface reflectance descriptors $f_{L}$, $f_{d} n_{2}$, and $f_{d} n_{3}$ are related to quantum catches by a square matrix $G(\mathbf{m})$ that depends on the SPD of the illuminant. Formula (9a) may be written more briefly as

$$
\mathbf{q}(\mathbf{x}) \simeq G(\mathbf{m}) \mathbf{p}(\mathbf{x}),
$$

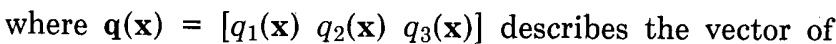
quantum catches as a function of position and where $\mathbf{p}(\mathbf{x})=$ $\left[f_{L}(\mathbf{x}) f_{d}(\mathbf{x}) n_{2} f_{d}(\mathbf{x}) n_{3}\right]$ describes surface reflectance at $\mathbf{x}$.

By applying the inverse $H(\mathbf{m})$ of the matrix $G(\mathbf{m})$ to both sides of formula $(9 \mathrm{~b})$, the illuminant is effectively discounted: if

$$
H(\mathbf{m})=G(\mathbf{m})^{-1},
$$

then 


$$
H(\mathbf{m}) \simeq \mathbf{p}(\mathbf{x})
$$

The principal implication of formula (11) is that information about surface reflectance can be abstracted by mechanisms whose sensitivities change appropriately in response to changes in the SPD of the illuminant. Such adaptive mechanisms cope best with surfaces and illuminants that are accurately represented as combinations of the three members of their respective basis sets. Moreover, the appropriate set of basis functions must be known by (or in some form built into) any mechanism that discounts the illuminant. Finally, the illuminant can be perfectly discounted only if its description $\mathbf{m}$ is known, a problem to which we now turn.

\section{FINDING AN UNKNOWN ILLUMINANT}

The simplest way to find the chromatic properties of a light source is to look directly at it. Another easy way is to look at its reflection in a mirror. Although the absolute intensity of the illuminant is not available in the reflected light, its chromaticity can be determined by a trichromatic system if the mirror is spectrally flat. For illuminants like daylight, which have SPD's that are fully described by the three parameters of intensity and chromaticity, finding the chromaticity alone is tantamount to finding the relative SPD. Several different methods can be used to estimate the chromaticity of the illuminant from reflections.

\section{Brightest Surface}

In the early retinex scheme of Land and McCann, the brightest region in a Mondrian display fulfills the function of a mirror. ${ }^{19,20}$ If the paper has a flat reflectance function one can obtain the chromaticity of the illuminant. Color constancy would therefore fail if there is no bright, white region in a scene.

\section{Specular Reflection (Highlights)}

Information from highlights can be used to estimate the chromaticity of the illuminant. Figure 3 shows that the lights from a uniformly colored object give rise to trichromatic responses that lie in the plane defined by axis $I$, which describes the specular component of reflectance, and the axis $D$, which describes the diffuse component. This plane is well defined only if both specular and diffuse components of reflectance contribute significant signals and vary across space in different ways. Such is generally the case for curved, glossy surfaces but not for flat, matte surfaces such as the papers in Mondrians.

If a scene contains two (or more) objects that are lit by the same illuminant and whose surfaces exhibit different variations in the two components of reflectance, then the chromaticity of the illuminant may be found by the means illustrated in Fig. 4. Because the specular component of reflection is common to both objects, their response planes intersect along the axis of the illuminant, as shown in Fig. 4A, and thus yield its chromaticity. This is easier to see in Fig. $4 \mathrm{~B}$, in which the response loci $L$ and $L^{\prime}$ have been projected onto the plane $C P$. The intersection of these lines in the chromaticity plane defines the chromaticity of the illuminant.

There are two substantial reasons to doubt that the visual system depends on such a method. First, to find the loci of responses that correspond to different objects, one must already have segmented the scene to establish which lights come from which objects. This begs the question of the purpose of color vision, which we believe plays an important role in the discrimination among objects and in their identification. Second, there is evidence that, at least under some circumstances, the visual system provides some degree of color constancy for scenes in which surfaces have constant saturations, e.g., in Mondrian displays in which the specular component of reflectance does not vary across an element. ${ }^{20}$

\section{Space Average}

If the average light reflected from the field of view has the chromaticity of the illuminant, then any mechanism that can sample the range of lights in a scene has access to the illuminant. In a uniformly illuminated scene, the spaceaveraged light bears the chromaticity of the illuminant if the space-averaged surface reflectance is flat. $8,21-23$

Does the average light in natural scenes bear the chromaticity of the illuminant? We know of no measurements of the space-averaged chromaticity in scenes illuminated by daylight. Although there are scenes in which the chromaticity of the average light departs significantly from that of the

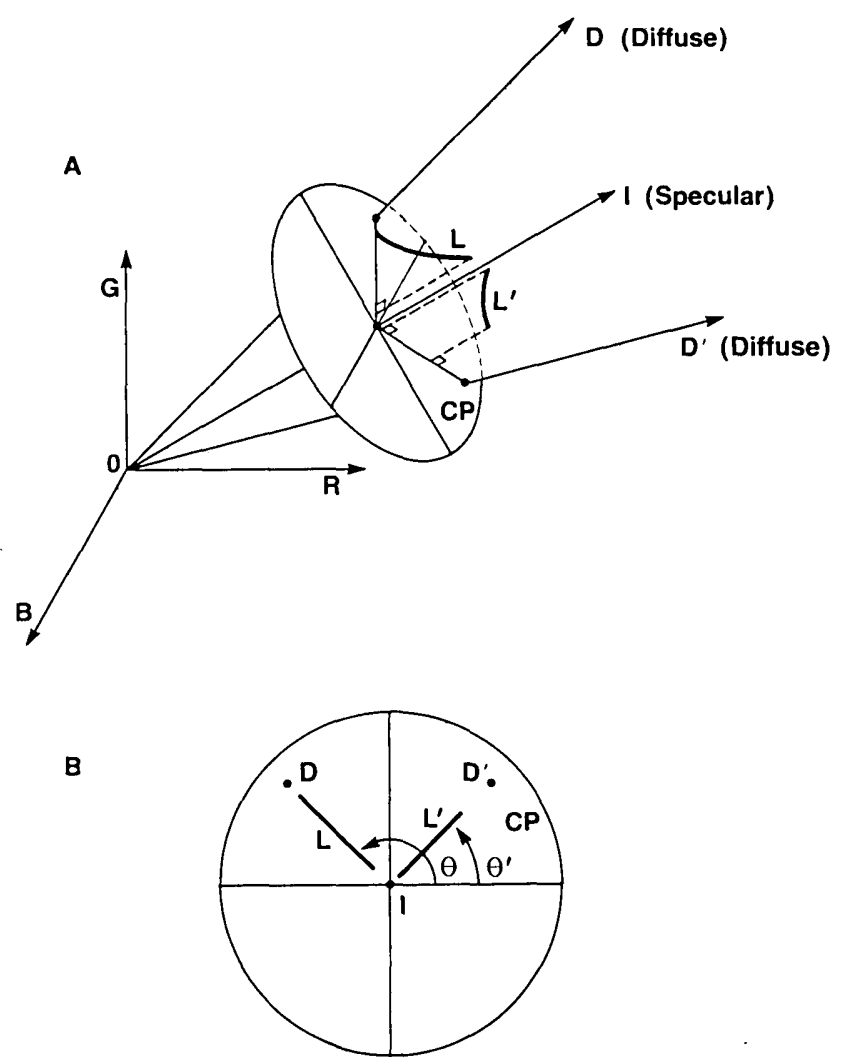

Fig. 4. A, Representation in the (R, G, B) space of responses $L$ and $L^{\prime}$ to two objects, lit by the same illuminant $I$, that have different diffuse components of reflectance $\left(D\right.$ and $\left.D^{\prime}\right)$. The two planes described by $L$ and $L^{\prime}$ intersect along the axis $I$, which describes the chromaticity of the illuminant, because the specular component of reflectance is common to both objects. The responses from two or more objects that define distinct planes can thus be used to find the axis $I$ that describes the chromaticity of the illuminant. B, Projection of $L$ and $L^{\prime}$ onto the chromaticity plane $C P$. The lines described by the responses intersect at the point $I$ marking the chromaticity of the illuminant. 
illuminant, we expect that the space-averaged light from most natural scenes will bear a chromaticity that closely approximates that of the illuminant.

The more interesting question, to which we now turn, is whether the human visual system relies on the average chromaticity's being that of the illuminant.

\section{MECHANISMS FOR DISCOUNTING THE ILLUMINANT}

Visual signals from a large number of distinct surfaces must be made available to mechanisms that recover the chromaticity of the illuminant. $8,9,24-26$ This does not mean that these mechanisms must be spatially extensive; the important requirement is that any point on the retina be able to sample the signals from a large part of the visual field. Mechanisms that are spatially localized have access to the space-averaged light from a scene through eye movements. Retinal mechanisms of adaptation can discount the spectral effects of the illuminant, provided that the space-averaged light, sampled through eye movements, has the chromaticity of the illuminant. The analysis proceeds by showing how the adjustments of retinal sensitivity made by known mechanisms of adaptation can bring about a transformation of the kind required by formulas (9)-(11).

\section{Scaled Cone Signals}

There is ample evidence from psychophysical experiments, ${ }^{27,28}$ now bolstered by physiological observations, ${ }^{29}$ that a multiplicative process regulates sensitivity independently in the three cone mechanisms, generally according to Weber's law.

Figure 5 shows the effects of this earliest stage of adaptation on the signaling of "light-dark" variations in surface reflectance for the reduced, two-dimensional case of $R$ - and $\mathrm{G}$-cone signals; the result is analogous for the three-dimensional case. On the left-hand side are shown the quantum catches that arise in R-and G-cones from a scene lit by either illuminant $I$ (top) or illuminant $I^{\prime}$ (bottom). Variations in the intensity of the illuminants describe the axes $I$ and $I^{\prime}$. These axes also describe variations in the weight on $S_{1}(\lambda)$ because $S_{1}(\lambda)$ is spectrally flat. The average quantum catches $\left(W_{R} W_{G}\right)$ and $\left(W_{R}^{\prime} W_{G}^{\prime}\right)$ arise from the product of the illuminant ( $I$ or $I^{\prime}$ ) and some amount $a$ of the reflectance $S_{1}(\lambda)$. Scaling quantum catches in R and $\mathrm{G}$ cones each by their average produces the cone signals $r$ and g; although the two axes $I$ and $I^{\prime}$ that describe the variations $f_{L}$ in lightness differ in the space of quantum catches (left), they coincide in the space of scaled cone signals (right).

\section{Recovering Relative Reflectance}

Scaling cone signals by their averages makes it hard to recover the absolute values of lightness $f_{L}$. Figure 5 shows how the average value of reflectance $a$, which can vary from scene to scene, always comes to lie in a fixed position in the space of scaled cone signals. There is no way to recover completely the description of surface reflectance $\mathbf{p}$ from scaled cone signals, unless the intensity of the illuminant or some absolute value of reflectance becomes known at some later stage in the visual system. The best that one can do without such information is to find a description of relative reflectance in which $\mathbf{p}$ is scaled by some unknown number $t$. This limitation affects any color-constancy scheme that uses reflections to find and discount the chromaticity of the illuminant.

Scaling the three terms $f_{L}, f_{d} n_{2}$, and $f_{d} n_{3}$ by some number $t$ does not, however, affect the computation of saturation or hue. The number $t$ disappears in the ratios that are formed to measure saturation and hue.

\section{Recovering Color}

Figure 6 shows how light adaptation in individual cone pathways affects the representation of variations in the contributions of the "red-green" and the "yellow-blue" basis functions to reflectance.

Consider again a scene that is lit by either illuminant $I$ or illuminant $I^{\prime}$. As shown on the left-hand side of Fig. 6, variations in the contributions of "red-green" and "yellowblue" basis functions to reflectance describe chromatic axes in the $(R, G, B)$ space of quantum catches. For $I$ these axes are given by varying the contributions of the lights $I S_{2}$ and $I S_{3}$ to the total light, while for $I^{\prime}$ the axes are determined by $I^{\prime} S_{2}$ and $I^{\prime} S_{3}$. The problem is that scaling cone signals, which can make the axes of the two illuminants coincide, cannot make the chromatic axes coincide, as shown on the right-hand side of Fig. 6. The reason for this lies in the relationship between surface reflectance and quantum catches described in formula (9a). Because any reasonable basis functions for illuminants and reflectances are spectrally broadband, the integrals that describe the link $G(\mathbf{m})$ between reflectance and quantum catches are typically nonzero. The three numbers used in scaling cone signals cannot undo what it takes nine numbers to describe.

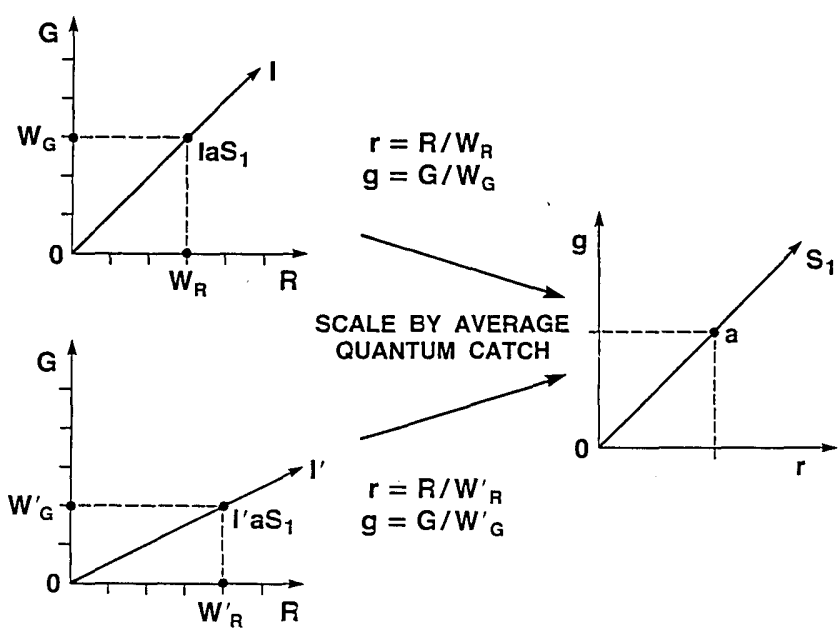

Fig. 5 The effect of scaling cone signals on the variations $f_{L}$ in the "light-dark" component $S_{1}$, shown for two dimensions of response. Illuminants $I$ and $I^{\prime}$ determine axes in the $(\mathrm{R}, \mathrm{G})$ space of quantum catches that describe variations in the contribution of $S_{1}$ to reflectance. If the average light bears the relative SPD of the illuminant, then the average light from the scene is some amount $a$ of the function $S_{1}$ multiplied by either $I$ or $I^{\prime}$. These average lights produce average quantum catch levels of $\left(W_{R} W_{G}\right)$ and $\left(W_{R}^{\prime} W_{G}^{\prime}\right)$, respectively; scaling cone quantum catches by these averages produces a space of scaled cone signals $(r, g)$, in which the axis that describes variations in $S_{1}$ is independent of the chromaticity of the illuminant. The average reflectance value $a$ comes to lie in a fixed position in $(\mathrm{r}, \mathrm{g})$ space: without knowledge of $a$ only relative values of reflectance may be recovered from scaled cone signals. 


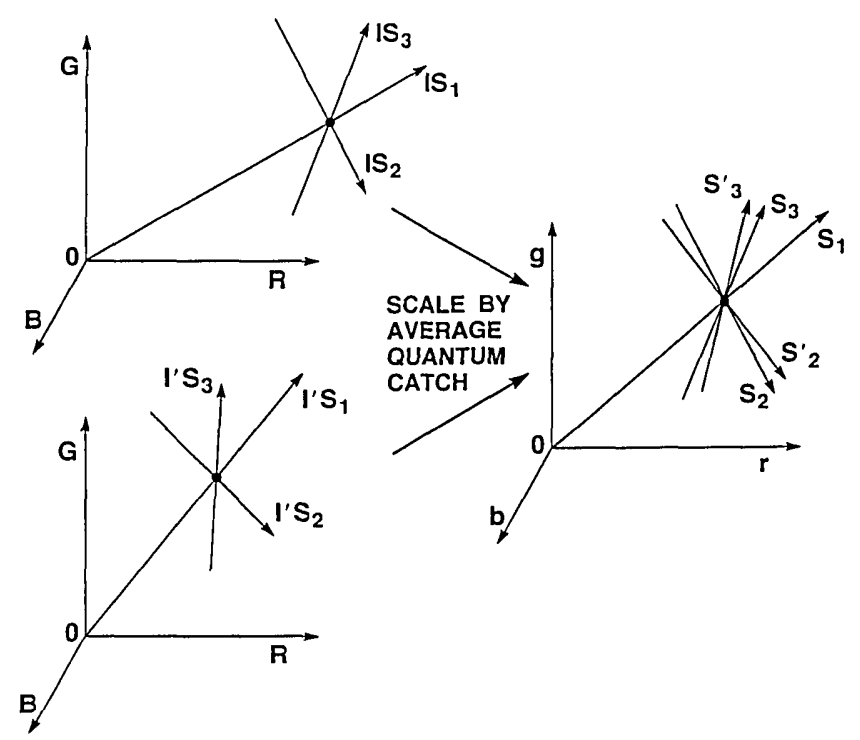

Fig. 6. Inability of simple scaling of cone signals to produce color constancy. Variations in the contributions of $S_{1}, S_{2}$, and $S_{3}$ to surface reflectance in a scene lit by illuminants $I$ or $I^{\prime}$ are shown in the ( $R, G, B$ ) space of quantum catches (left) and in the (r, g, b) space of scaled cone signals (right). Although scaling by average quantum catches can make the axes $I S_{1}$ and $I^{\prime} S_{1}$ coincide along axis $S_{1}$ in $(\mathrm{r}, \mathrm{g}, \mathrm{b})$ space, scaling will not make coincident the axes that describe the contributions of $S_{2}$ and $S_{3}$ to reflectance.

Color-opponent mechanisms that are set to abstract variations in $S_{2}(\lambda)$ and $S_{3}(\lambda)$ from scaled cone signals under one illuminant will fail if the illuminant is changed unless there is a second stage of adaptation that can adjust their sensitivities appropriately. Formulas (9)-(11) show that to abstract the "color" terms we need two color-opponent mechanisms that combine scaled cone signals linearly with weights that vary differently with the state of adaptation.

\section{Formal Expression for Discounting the Chromaticity of} the Illuminant

The second stage of adaptation needed to discount the residual effects of the illuminant in scaled cone signals may be expressed by modifying formula (9b) to incorporate the effects of scaling. We can scale photoreceptoral quantum catches by the average quantum catch $a\left\langle I(\mathbf{m}) S_{1}, Q_{k}\right\rangle$ by applying the matrix $D(\mathbf{m})$ to the vector $\mathbf{q}(\mathbf{x})$ of quantum catches, where $D(\mathbf{m})$ is given by

$D(\mathbf{m}) \simeq$

$$
\left[\begin{array}{ccc}
1 / a\left\langle I(\mathbf{m}) S_{1}, Q_{1}\right\rangle & 0 & 0 \\
0 & 1 / a\left\langle I(\mathbf{m}) S_{1}, Q_{2}\right\rangle & 0 \\
0 & 0 & 1 / a\left\langle I(\mathbf{m}) S_{1}, Q_{3}\right\rangle
\end{array}\right]
$$

Applying the diagonal matrix $D(\mathbf{m})$ to both sides of formula (9b), we have

$$
D(\mathbf{m}) \mathbf{q}(\mathbf{x}) \simeq D(\mathbf{m}) G(\mathbf{m}) \mathbf{p}(\mathbf{x}) .
$$

Because the inverse of a diagonal matrix is itself diagonal, the matrix $D(\mathbf{m})$ is not the inverse of the matrix $G(\mathbf{m})$, and so the multiplicative scaling of cone signals (left) cannot completely discount the illuminant.

Formula (13) indicates that to recover the description of reflectance $\mathbf{p}(\mathbf{x})$, the inverse $E(\mathbf{m})$ of the product $D(\mathbf{m}) G(\mathbf{m})$ must be applied to scaled cone signals: if

$$
E(\mathbf{m})=[D(\mathbf{m}) G(\mathbf{m})]^{-1},
$$

then

$$
E(\mathbf{m}) D(\mathbf{m}) \mathbf{q}(\mathbf{x}) \simeq \mathbf{p}(\mathbf{x}) .
$$

Because the average value of reflectance $a$ is assumed to be unknown, we take the second stage $E(\mathbf{m})$ to be implemented by an otherwise identical matrix $E^{\prime}(\mathbf{m})$ with terms that have been scaled by some constant. The two stages $D(\mathbf{m})$ and $E^{\prime}(\mathbf{m})$ of adaptation thus recover a relative description of reflectance $t \mathbf{p}(\mathbf{x})$.

\section{Second Sites of Adaptation}

Two major lines of psychophysical evidence point to the existence of second-stage adaptive mechanisms that act after the multiplicative scaling in the individual cone pathways. The first of these come from experiments on what Wyszecki and Stiles call "asymmetric matching"-matching the appearance of lights viewed under one state of chromatic adaptation to lights viewed under a different state of chromatic adaptation. ${ }^{11}$ Color matches made across different states of adaptation cannot be explained by the hypothesis (due to Von $\mathrm{Kries}^{30}$ ) that the sensitivity of the visual system is regulated solely by multiplicative processes in the individual cone mechanisms. ${ }^{11,31}$

The second line of evidence comes from experiments in which Stiles's two-color increment threshold technique has been used to study the spectral sensitivities of the mechanisms that regulate sensitivity to background lights. ${ }^{28,32-37}$ These experiments have revealed adaptive mechanisms that appear to be driven by opposed signals from different classes of cone. ${ }^{32-37}$

Evidence from electroretinography points to the existence of second sites of adaptation within the retina, distal to the ganglion cells. ${ }^{38}$ Single-unit recordings from the macaque's retina and lateral geniculate nucleus (LGN) reveal two classes of chromatically opponent neurons, whose preferred chromaticities fall broadly along either a "red-green" axis or a "blue-yellow" axis. ${ }^{39-42}$ It is therefore natural to suppose that these mechanisms convey the terms $f_{d} n_{2}$ and $f_{d} n_{3}$, from which stable hue descriptors may be derived.

\section{Adaptation to the Space-Averaged Light}

Mechanisms of light adaptation (both in the separate cone pathways and at color-opponent sites) can discount the illuminant only if they adjust sensitivity over a period long enough to ensure that the space-averaged light, accumulated through the normal pattern of eye movements used in viewing a scene, regulates the adjustment.

The time course of light adaptation has been assessed in several experiments, typically by measuring the threshold for detection of a brief flash at various times after a step change in the illuminance of a steady background light. Although a considerable change in sensitivity occurs during the first $200 \mathrm{msec}$ following the change in background, ${ }^{43,44} \mathrm{a}$ stable state may be reached much more slowly. This is particularly evident in observations that appear to tap the adaptation of second-stage mechanisms. The sensitivity of the $\Pi_{1}$ mechanism, which is believed to be regulated at an opponent site, ${ }^{34}$ changes slowly following either an incre- 
ment ${ }^{33}$ or a decrement ${ }^{32}$ in background illumination; indeed, some observations ${ }^{35}$ suggest that the adaptive state of the second site may stabilize only after several hundred seconds.

Eye, head, and body movements serve to expose small regions of retina to many parts of a scene over a short period: typical fixations in the viewing of a complex scene last from 0.25 to $0.50 \mathrm{sec}^{45}$ Active scanning of a scene may thus ensure that the adaptive state at any point on the retina reflects the space-averaged quantal absorption rate. In this steady state of adaptation, a stimulus falling on a particular retinal region will evoke a response that depends on the degree to which the chromaticity and intensity of the stimulus differ from those to which that region has become adapted.

\section{CENTRAL REPRESENTATION OF THE HUE DESCRIPTOR}

We have shown how two stages of adaptation, coupled with eye movements, can discount the illuminant. The output of second-stage chromatic mechanisms in a steady state of adaptation can provide a scaled representation of surface reflectance. We now consider how signals from retinal ganglion cells might be combined by central mechanisms to provide a representation of hue.

\section{Spatiochromatic Opponency}

Physiological recordings from single neurons in the primate's retina and LGN show that cells of the slow conducting (parvocellular) type have chromatically opponent receptive fields and are excited by lights that have a limited range of chromaticities. ${ }^{39-42,46,47}$ When in a steady state of light adaptation, these neurons combine linearly the signals that arise in the different classes of cone. ${ }^{46,47}$

This linear behavior allows us to represent the chromatic sensitivity of a cell in a three-dimensional chromaticity space by a vector $\mathbf{G}$ drawn from the "white point" $W$ that represents the steady state of adaptation; see Fig. 7. This vector $\mathbf{G}$ represents the best stimulus to use in stimulating the cell and is the cell's response gradient. ${ }^{48}$ The preferred hue $\theta_{G}$ for a cell is found by projecting its gradient vector onto the chromaticity plane.

Ganglion cells and neurons in the LGN lack an attribute that might be thought important for mechanisms that provide hue constancy. Because the chromatically opponent mechanisms are separated in the center and surround of the receptive field, the responses of these neurons depend on both the spatial and chromatic properties of the stimulus. ${ }^{47,49}$ As spatial frequency is raised, the cells respond better to achromatic contrast, so the gradient vectors that represent the chromatic signatures of such cells rotate away from the chromaticity plane. The responses of such units therefore cannot be used directly to estimate hue because they confound variations in lightness with variations in chromaticity in a space-dependent manner. To obtain geometrically stable hue descriptors we need mechanisms that have gradients with fixed directions in the chromaticity plane.

\section{Labeled Lines for Hue}

The hue descriptor is represented by the ratio $n_{3} / n_{2}$, which may be thought of as the ratio of signals in the "red-green" and "blue-yellow" opponent pathways. We think it unlikely that any mechanism signals hue by taking this ratio directly because ratios are not well behaved. A more plausible scheme is that hue is represented not by the size of the response of a neuron but by the identification of which neuron responds best. This idea of "labeled lines" leads us to think of a population of neurons tuned to different azimuths; the hue of the stimulus is established by finding the most active neuron.

Figure 8 shows how the linear combination of signals from spatiochromatically opponent units can give rise to chromatic mechanisms with gradient vectors that retain their directions through changes in the spatial properties of the stimulus. The strength of the gradient representation is that gradients are additive, and so a cell that linearly combines the signals from two or more cells will have a gradient that is the same linear combination of these cells' gradients. One eliminates responses to achromatic stimuli by summing responses of appropriate pairs of color-opponent cells that have identical locations and receptive-field space constants. Opposing tendencies to respond to achromatic stimuli are canceled through such addition.

The result of such selective combination is cells that at all spatial frequencies respond best to purely chromatic modulations in a steady state of adaptation. Linear combinations of these units in turn result in mechanisms that respond maximally to any desired hue. Evidence from unpublished physiological experiments by Lennie, Sclar, and Krauskopf suggests that in the striate cortex of the macaque monkey

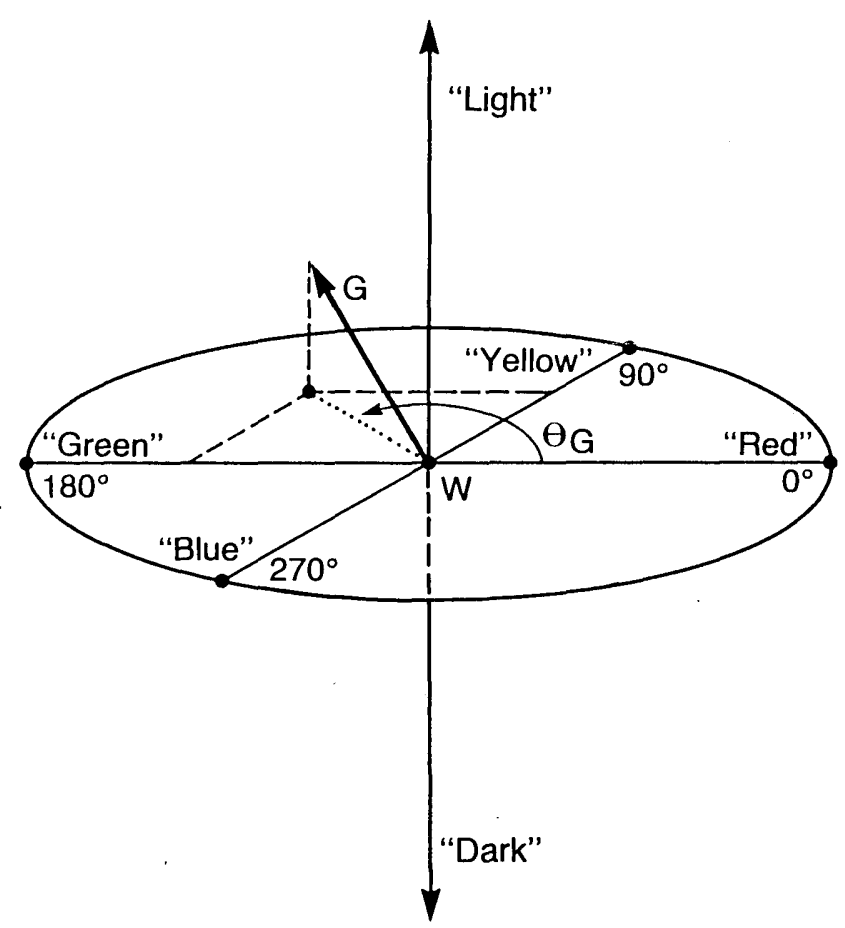

Fig. 7. Color space in which can be represented the chromatic sensitivity of a cell adapted to some steady light represented by the "white point" $W$. The sensitivity of a cell that combines cone signals linearly may be represented by a vector $\mathbf{G}$, its response gradient. The vector $\mathbf{G}$ represents the most effective choice of chromatic contrasts to use in stimulating the cell. The preferred hue $\theta_{G}$ of the cell may be found by projecting $\mathbf{G}$ onto the chromaticity plane. 

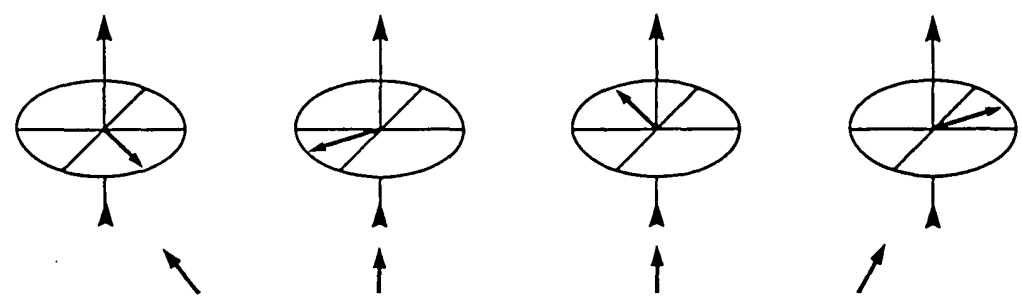

$L \quad I N E A R$

C $\mathrm{O}$
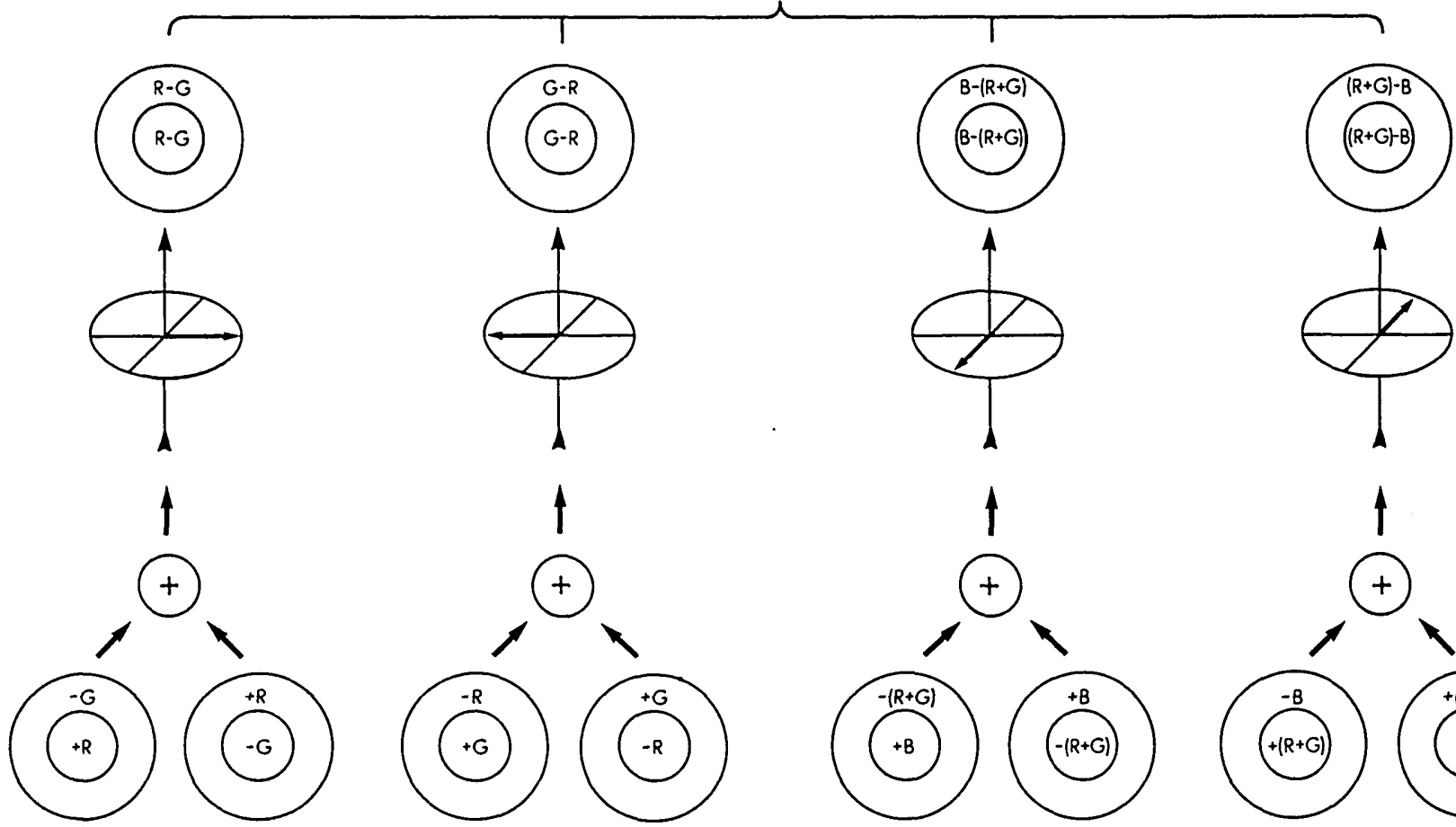

$\uparrow$
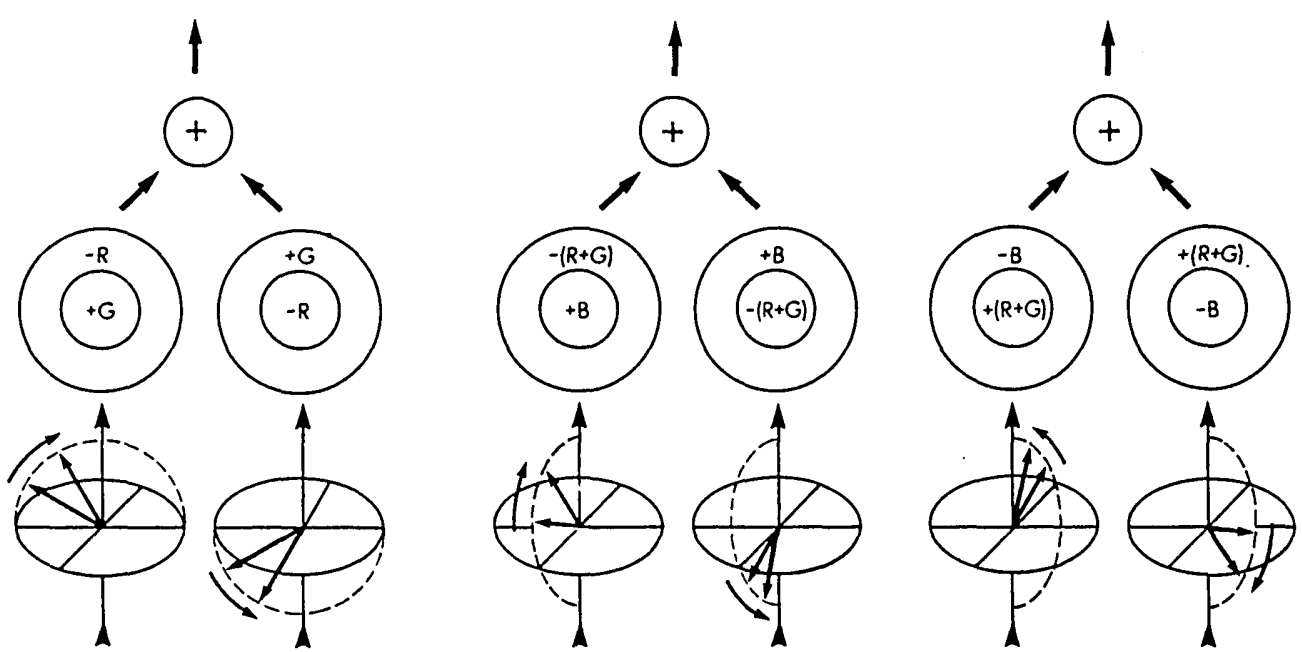

Fig. 8. Gradient representation of successive stages in the analysis of chromatic signals. The most effective stimuli for chromatically opponent units in the retina and parvocellular layers of the LGN (bottom row) are represented in the color space of Fig. 7. Arrows show that the best stimuli become increasingly achromatic as spatial frequency is increased. Opposing tendencies to respond to achromatic stimuli are canceled by addition of signals from appropriate pairs of units. The result (middle row) is mechanisms that at all spatial frequencies respond best to purely chromatic modulations about $W$. Linear combinations of signals from these units, in turn, result in mechanisms that respond maximally to any desired hue (top row).

the preferred azimuths of neurons span a wide range. These units may be the basis for an azimuthal description of hue.

\section{SUMMARY AND IMPLICATIONS}

Our first goal has been to provide a quantitative account of how one can find the colors of solid objects regardless of their shapes or positions and regardless of the chromaticity of the illuminant. We recover three descriptors of surface reflectance from each point in a scene, from which are derived hue descriptors that are independent of geometric variables. Our scheme does not require that the scene be segmented before chromatic descriptors are extracted; indeed, the geo. metric stability of hue permits its use in segmenting a scene, and the variations in specular and diffuse components of reflection carried along the dimensions of lightness and saturation permit their use in establishing an object's shape and position.

Our formal analysis assumes that the SPD of the illuminant is known, but in any physiologically plausible account of color constancy this knowledge is unlikely to be represented explicitly. Our second goal has therefore been to provide an account of how known mechanisms of the human visual system might discount the illuminant. We have shown that if the space-averaged light from a scene bears the relative SPD of the illuminant, then two stages of light adaptation (a multiplicative change in sensitivity in the independent cone mechanisms followed by an adaptive linear transformation of scaled cone signals at color-opponent sites), when coupled with eye movements that expose them to the average light, 
yield signals in second-stage mechanisms from which hue descriptors can easily be derived.

\section{The Unknown Illuminant}

Second-stage mechanisms that are in a steady state of adaptation provide good estimates of surface reflectance only if the average chromaticity of a scene is the chromaticity of the illuminant. Our scheme suggests that the more a scene departs from this condition, the poorer the estimates of surface reflectance provided by the retina will be.

Buchsbaum ${ }^{8}$ suggests that the visual system uses another means to find the illuminant: the space-averaged surface reflectance is known and is not necessarily flat. Buchsbaum's method has difficulty handling a collection of surfaces with unknown properties but may be a useful means of establishing the SPD of the illuminant when the visual environment does not meet the conditions needed for the postulated mechanisms of adaptation to provide color constancy (for example, a room whose surfaces have a strongly predominant color). In this case, knowledge of the surface properties of objects might be used to correct biases in the signals generated by second-stage mechanisms.

Another alternative to the assumption that the average chromaticity is that of the illuminant is to assume, as have Maloney and Wandell, ${ }^{9}$ that surface reflectances encountered by a trichromatic visual system are described adequately by only two parameters. In this case no further assumption about surface reflectances need be made, but the penalty is less robust constancy of color appearance.

\section{Physiological Mechanisms}

Our account leaves unresolved several questions about the estimates of surface reflectance made by chromatically opponent neurons. We do not know that ganglion cells incorporate (implicitly) the basis functions required to render adequate descriptions of natural surfaces. Furthermore, adaptation at color-opponent sites is required to adjust the sensitivity of a neuron so that it signals a constant reflectance descriptor; the range of sensitivities required suggests that opponent mechanisms must receive inputs from all three classes of cone. Although some chromatically opponent ganglion cells and neurons in the LGN receive inputs from all three classes of cone, when adapted to white light most show no discernible input from $B$ cones. ${ }^{46,47}$ However a change in the chromaticity to which a cell is adapted might reveal otherwise hidden inputs from $B$ cones (a possibility for which DeMonasterio et al. ${ }^{42}$ provide some evidence).

Alternatives to the postulated second stage of adaptation within the retina are that adaptation may occur in cortex, where we know that many color-opponent neurons receive inputs from all three classes of cone, or that the adjustments to scaled cone signals made by our second stage are not actually made by the visual system. At the moment too little is known about adaptation at second sites (especially adaptation brought about by modest chromatic excursions from white) to be sure that the mechanisms so far revealed have the properties that we require.

\section{Physical Limitations}

Our model of lights from surfaces assumes several conditions that need not be true of the real world. The illuminant must be spatiochromatically homogeneous, and its SPD must be adequately represented by weights on a particular set of three basis functions that characterize daylight. We therefore expect color constancy to be impaired if the illuminant is heterogeneous (e.g., when objects in a room with a window are illuminated by daylight from an overcast sky and also by a tungsten source) or if its SPD departs markedly from the range represented by the phases of daylight (e.g., when it is composed of one, two, or even three spectrally narrow-band sources). Moreover, we have taken scenes to be shadowless and have assumed negligible interreflectance among surfaces. We expect the colors of shadowed objects to be less constant than those of objects lit directly. Finally, color constancy should be poorer for surfaces whose reflectances cannot be described accurately by the three basis functions built into the visual system or whose reflectances cannot simply be divided into specular and diffuse components (e.g., fluorescent and iridescent surfaces).

\section{ACKNOWLEDGMENTS}

This work was supported by National Institutes of Health grants EY-04440 and EY-01319. M. D'Zmura held an IBM graduate fellowship.

\section{REFERENCES}

1. D. B. Judd, D. L. MacAdam, and G. Wyszecki, "Spectral distribution of typical daylight as a function of correlated color temperature,"J. Opt. Soc. Am. 54, 1031-1040 (1964).

2. E. R. Dixon, "Spectral distribution of Australian daylight," J. Opt. Soc: Am. 68, 437-450 (1978).

3. J. Cohen, "Dependency of the spectral reflectance curves of the Munsell color chips," Psychon. Sci. 1, 369-370 (1964).

4. L. T. Maloney, "Computational approaches to color constancy," Applied Psychology Lab. Tech. Rep. 1985-01 (Stanford University, Stanford, Calif., 1985).

5. P. Sällström, "Colour and physics: some remarks concerning the physical aspects of human colour vision," Institute of Physics Rep. 73-09 (University of Stockholm, Sweden, 1973).

6. M. H. Brill, "A device performing illuminant-invariant assessment of chromatic relations," J. Theor. Biol. 71, 473-478 (1978).

7. M. H. Brill, "Further features of the illuminant-invariant trichromatic photosensor," J. Theor. Biol. 78, 305-308 (1979).

8. G. Buchsbaum, "A spatial processor model for object colour perception," J. Franklin Inst. 310, 1-26 (1980).

9. L. T. Maloney and B. A. Wandell, "Color constancy: a method for recovering surface spectral reflectance," J. Opt. Soc. Am. A 3, 29-33 (1986).

10. H. Yilmaz, "Color vision and a new approach to general perception," in Biological Prototypes and Synthetic Systems, E. E. Bernard and M. R. Kare, eds. (Plenum, New York, 1962).

11. G. Wyszecki and W. S. Stiles, Color Science. Concepts and Methods, Quantitative Data and Formulae (Wiley, New York, 1982).

12. K. E. Torrance and E. M. Sparrow, "Theory of off-specular reflection from roughened surfaces," J. Opt. Soc. Am. 57, 11051114 (1967).

13. T. S. Trowbridge and K. P. Reitz, "Average irregularity representation of a rough surface for ray reflection," J. Opt. Soc. Am. 65, 531-536 (1975).

14. B. T. Phong, "Illumination for computer generated pictures," Commun. ACM 18, 311-317 (1975).

15. J. N. Lythgoe, The Ecology of Vision (Clarendon, Oxford, 1979).

16. K. Nassau, The Physics and Chemistry of Color; The Fifteen Causes of Color (Wiley, New York, 1983).

17. S. A. Shafer, "Using color to separate reflection components," Department of Computer Sciences Tech. Rep. 136 (University of Rochester, New York, 1984).

18. V. C. Smith and J. Pokorny, "Spectral sensitivity of the foveal 
cone photopigments between 400 and $500 \mathrm{~nm}$," Vision Res. 15, 161-171 (1975).

19. E. H. Land and J. J. McCann, "Lightness and retinex theory," J. Opt. Soc. Am. 61, 1-11 (1971).

20. J. J. McCann, S. P. McKee, and T. H. Taylor, "Quantitative studies in retinex theory: a comparison between theoretical predictions and observer responses to the 'color mondrian' experiments," Vision Res. 16, 445-458 (1976).

21. H. Helson, "Fundamental problems in color vision. I. The principle governing changes in hue, saturation and lightness of non-selective samples in chromatic illumination," J. Exp. Psychol. 23, 439-476 (1938).

22. D. B. Judd, "Hue saturation and lightness of surface colors with chromatic illumination," J. Opt. Soc. Am. 30, 2-32 (1940).

23. H. Helson, D. B. Judd, and M. H. Warren, "Object-color changes from daylight to incandescent filament illumination," Illum. Eng. 47, 221-232 (1952).

24. E. H. Land, "The retinex theory of color vision," Sci. Am. 237, 108-128 (1977).

25. E. H. Land, "Recent advances in retinex theory and some implications for cortical computations: color vision and the natural image," Proc. Natl. Acad. Sci. USA 80, 5163-5169 (1983).

26. E. H. Land, "Recent advances in retinex theory," Vision Res. 26, 7-21 (1986).

27. W. S. Stiles, "Increment thresholds and the mechanisms of colour vision," Doc. Ophthalm. 3, 138-163 (1949).

28. W. S. Stiles, Mechanisms of Colour Vision (Academic, New York, 1978)

29. J. M. Valeton and D. van Norren, "Light adaptation of primate cones: an analysis based on extracellular data,"Vision Res. 23, 1539-1547 (1983).

30. H. von Helmholtz, Treatise on Physiological Optics, J. P. C. Southall, ed. (Dover, New York, 1962), Vol. II.

31. D. Jameson and L. M. Hurvich, "Color adaptation: sensitivity, contrast, after-images," in Handbook of Sensory Physiology, D. Jameson and L. M. Hurvich, eds. (Springer-Verlag, New York, 1972), Vol. VII/4.

32. J. D. Mollon and P. G. Polden, "An anomaly in the response of the eye to light of short wavelengths," Phil. Trans. R. Soc. London Ser. B 278, 207-240 (1977).

33. E. J. Augenstein and E. N. Pugh, "The dynamics of the $\Pi_{1}$ colour mechanism: further evidence for two sites of adaptation," J. Physiol. 272, 247-281 (1977).

34. E. N. Pugh and J. D. Mollon, "A theory of the $\Pi_{1}$ and $\Pi_{3}$ colour mechanisms of Stiles," Vision Res. 19, 293-312 (1979).
35. J. M. Loomis, "Transient tritanopia: failure of time-intensity reciprocity in adaptation to long-wavelength light," Vision Res. 20, 837-846 (1980).

36. B. A. Wandell and E. N. Pugh, "A field-additive pathway detects brief-duration, long-wavelength incremental flashes," Vision Res. 20, 613-624 (1980).

37. B. A. Wandell and E. N. Pugh, "Detection of long-duration incremental flashes by a chromatically coded pathway," Vision Res. 20, 625-635 (1980).

38. J. M. Valeton and D. van Norren, "Transient tritanopia at the level of the erg $b$-wave," Vision Res. 19, 689-693 (1979).

39. R. L. DeValois, I. Abramov, and G. H. Jacobs, "Analysis of response patterns of LGN cells," J. Opt. Soc. Am. 56, 966-977 (1966).

40. T. N. Wiesel and D. H. Hubel, "Spatial and chromatic interactions in the lateral geniculate body of the rhesus monkey," J. Neurophysiol. 29, 1115-1156 (1966).

41. F. M. DeMonasterio and P. Gouras, "Functional properties of ganglion cells of the rhesus monkey retina," J. Physiol. 251, 167195 (1975).

42. F. M. DeMonasterio, P. Gouras, and D. J. Tolhurst, "Concealed colour opponency in ganglion cells of the rhesus monkey retina," J. Physiol: 251, 217-229 (1975).

43. B. H. Crawford, "Visual adaptation in relation to brief conditioning stimuli," Proc. R. Soc. London Ser. B 134, 283-302 (1947).

44. M. M. Hayhoe, N. Benimoff, and D. C. Hood, "The time course of multiplicative and subtractive adaptation processes," Vision Res. (to be published).

45. A. L. Yarbus, Eye Movements and Vision, B. Haigh, translator, L. A. Riggs, ed. (Plenum, New York, 1967).

46. C. C. A. M. Gielen, J. A. M. Van Gisbergen, and A. J. H. Vendrik, "Reconstruction of responses of colour-opponent neurones in monkey lateral geniculate nucleus," Biol. Cybern. 44, 211-221 (1982).

47. A. M. Derrington, J. Krauskopf, and P. Lennie, "Chromatic mechanisms in lateral geniculate nucleus of macaque," J. Physiol. 357, 241-265 (1984).

48. B. O'Neill, Elementary Differential Geometry (Academic, New York, 1966).

49. C. R. Ingling, Jr. and E. Martinez-Uriegas, "The relationship between spectral sensitivity and spatial sensitivity for the primate r, g x-channel," Vision Res. 23, 1495-1500 (1983). 